\title{
Improved Empirical Identification of the Inverse Model of a Squeeze-Film Damper Bearing based on a Recurrent Neural Network
}

DOI:

10.1115/gt2018-75777

\section{Document Version}

Accepted author manuscript

Link to publication record in Manchester Research Explorer

Citation for published version (APA):

Al-Ghazal, G. G., Bonello, P., \& Torres Cedillo, S. G. (2018). Improved Empirical Identification of the Inverse Model of a Squeeze-Film Damper Bearing based on a Recurrent Neural Network. In Proceedings of ASME Turbo Expo 2018: Turbomachinery Technical Conference and Exposition https://doi.org/10.1115/gt2018-75777

\section{Published in:}

Proceedings of ASME Turbo Expo 2018: Turbomachinery Technical Conference and Exposition

\section{Citing this paper}

Please note that where the full-text provided on Manchester Research Explorer is the Author Accepted Manuscript or Proof version this may differ from the final Published version. If citing, it is advised that you check and use the publisher's definitive version.

\section{General rights}

Copyright and moral rights for the publications made accessible in the Research Explorer are retained by the authors and/or other copyright owners and it is a condition of accessing publications that users recognise and abide by the legal requirements associated with these rights.

\section{Takedown policy}

If you believe that this document breaches copyright please refer to the University of Manchester's Takedown Procedures [http://man.ac.uk/04Y6Bo] or contact uml.scholarlycommunications@manchester.ac.uk providing relevant details, so we can investigate your claim.

\section{OPEN ACCESS}




\title{
Improved Empirical Identification of the Inverse Model of a Squeeze-Film Damper Bearing based on a Recurrent Neural Network
}

\author{
Ghaith Ghanim Al-Ghazal \\ School of Mechanical, Aerospace and Civil \\ Engineering, University of Manchester, \\ Manchester, United Kingdom
}

\author{
Philip Bonello \\ School of Mechanical, Aerospace and Civil \\ Engineering, University of Manchester, \\ Manchester, United Kingdom
}

\author{
Sergio G. Torres Cedillo \\ Centro Tecnológico Aragón, FES \\ Aragón, Universidad Nacional \\ Autónoma de México (UNAM), \\ Av. Rancho Seco s/n, Edo. de \\ México, México. C.P. 57130
}

\begin{abstract}
Most recently proposed techniques for inverse rotordynamic problems seek to identify the unbalance on a rotor using a known structural model and measurements from externally mounted sensors only. Such non-intrusive techniques are important for balancing rotors that cannot be accessed under operational conditions because of temperature or space restrictions. The presence of nonlinear bearings, like squeezefilm damper (SFD) bearings used in aero-engines, complicates the solution process of the inverse rotordynamic problem. In certain practical aero-engine configurations, the solution process requires a substitute for internal instrumentation to quantify the SFD journal vibration. This can be provided by an inverse model of the SFD bearing which outputs the time history of the relative vibration of the SFD journal relative to its housing, for a given input time history of the SFD force. This paper focuses on the inverse model of the SFD and presents an improved methodology for its identification via a Recurrent Neural Network (RNN) trained using experimental data from a purposely designed rig. The novel application of chirp excitation via two orthogonal shakers considerably improves both the quality of the training data and the efficiency of its generation, relative to an earlier preliminary work. Validation test results show that the RNNs can predict the journal displacement time history with reasonable accuracy. It is therefore expected that such an inverse SFD model would serve as a reliable component in the solution of the wider inverse problem of a rotordynamic system.
\end{abstract}

\section{INTRODUCTION}

In rotordynamic analysis, the prediction of the dynamic response to a given unbalance distribution is referred to as the forward problem [1-4]. The bearing models used in the solution process of forward problems provide the bearing forces for given instantaneous displacements/velocities of the journal (inner member) relative the housing. Such bearing models can themselves be referred to as forward bearing models, where the force is the output and displacement/velocity is the input [5]. Advances in modeling capability, including nonlinear SFD bearing models, enable effective solution of the forward problem. However, as observed by Peters et al [1], in a practical situation the true unbalance distribution is not known. Moreover, a state of unbalance can develop in service. Hence, the inverse problem, that seeks to determine the unbalance from measurements, is vital for monitoring changes to the state of balance of the engine over its operational lifetime and correct (balance) it as necessary [1-7]. A considerable number of research works have been devoted to the solution of the inverse problem using a known structural model and measurements from externally mounted sensors only (e.g. accelerometers on bearing pedestals or aero-engine casings) [1-7]. The motivation for using a non-intrusive inverse problem solution approach over conventional 'trial mass' balancing is the need for a "right first time" approach towards the balancing of rotors that are inaccessible under operational conditions due to space and temperature restrictions, as is the case for the high pressure rotor of an aero-engine [2,3]. Such problems are complicated by nonlinearity in the bearings, particularly in the case of SFD bearings, where the nonlinear effect of the oil annulus surrounding the outer race of the rolling element bearing cannot be simplified without incurring significant error [2]. 
As observed in [1-5], most research on inverse problems in rotordynamics has been limited by the assumption of bearing linearity e.g. $[1,3,6,7]$. Work on the solution of nonlinear inverse problems in rotordynamics was initiated by Dicken et al. [2] but not continued until the recent works by TorresCedillo and Bonello [4,5]. The solution of the inverse rotordynamic problem considered by Dicken et al. [2] required an inverse bearing model that relates the relative displacement of the SFD journal relative to the housing (input) to the SFD force (output). However, this database was severely limited since it was based on a theoretical model that assumes that the bearing force could be expressed as the product of an amplitude-dependent coefficient and the vibration. From basic SFD theory [8], this theoretical model is only valid for the case of uncavitated SFDs with journals undergoing circular orbits that are concentric with the circular clearance bore. Apart from being highly idealized, this assumption limited the full inverse problem solved by Dicken et al. [2] to isotropic conditions and their work was not experimentally validated.

The research by Torres-Cedillo and Bonello [4] has shown that the full inverse problem of a nonlinear system can be solved without the need of an inverse bearing model provided there is at least one linear connection between the rotor (source of the unbalance) and the casing (measurement positions), apart from the nonlinear connections (as indeed was the case for the problem considered by Dicken et al. [2]). However, if there is only one linear connection and this connection is weak (e.g. a soft retainer spring in parallel with the SFD), or no linear connection at all, the solution process requires a means to quantify the SFD journal vibration [5]. This information can be provided by an inverse model of the SFD bearing. Provided such a model is more realistic than the database used by Dicken et al. [2], it can then be used as a substitute for internal instrumentation within the inverse rotordynamic problem that determines the unbalance using externally mounted sensors.

The focus of this paper is the identification of the inverse model of the SFD bearing, not its use within the wider inverse rotordynamic problem. The output of the inverse model of the SFD is the vibration of the SFD journal relative to its housing and its input is the SFD force. For the identification of both forward and inverse SFD models, due to the complex nonlinearity of the SFD, a non-parametric (i.e. "black box") approach for identification is preferred [5,9], since this does not make any assumptions on the nature of the underlying relationship between input and output. Moreover, it has the ability of capturing physical complexities that are difficult to model e.g. irregularities in the SFD radial clearance. However, the identification of the inverse model is fundamentally different from that of the forward model due to the need to account for the time history of the inputs and outputs in the inverse model. According to the Reynolds Equation of an incompressible fluid, the instantaneous SFD bearing force is a function of the instantaneous displacements/velocities. Hence, in the case of the forward model (where the input is the SFD journal motion and the output is the force) a simple "memoryless" non-parametric identification technique based on Chebyshev polynomial fitting [10-12] or static neural networks [9] can be used. No such instantaneous mapping (function) exists if the input and output are switched, but Torres Cedillo and Bonello [5] showed that the inverse SFD model can be reliably identified using a Recurrent Neural Network (RNN), which outputs the instantaneous SFD journal displacement given the time history of the bearing force and the time history of the estimated SFD journal displacement output which is fed back to the network. The RNN used to identify the nonlinear inverse model of the SFD was trained using experimental data from a specially designed SFD test rig wherein the whirl motion of the journal within the oil-filled clearance was induced by two orthogonal shakers [5]. Torres-Cedillo and Bonello [5] then used the identified inverse SFD model within the solution of the inverse problem of the simple non-rotating experimental rig, in order to identify the amplitudes and phases of the orthogonal excitation forces on the shaft, using only accelerometer readings at the bearing housing.

One significant limitation of the method used by Torres Cedillo and Bonello [5] was the need for many empirical input-output data sets to be employed for training in order to cover a wide range of rotor responses. The training data in [5] were constructed by concatenating a random combination of 10 cycles each of (discrete frequency, discrete amplitude) sinusoidal signals that were measured to cover all cases from $10-150 \mathrm{~Hz}$ excitation frequencies (at $10 \mathrm{~Hz}$ intervals) with force amplitude from 25-150 N (at $10 \mathrm{~N}$ intervals).

The contribution of the present paper is the experimental implementation of a novel methodology for training a RNN for the identification of the inverse SFD model using data generated in a single run of a continuous frequency sweep (chirp excitation). The idea was originally proposed by Bin Hassan and Bonello [13] for the identification of foil-air bearings but was not implemented experimentally. The proposed method is motivated by the following advantages:

- Saves laboratory time;

- Decreases size of training data, which reduces the training time;

- Enhances the prediction performance of the vibration response.

Using empirical data from a purposely designed test rig, appropriately designed and trained RNNs are presented to reproduce the input/output relationship governing the inverse SFD model over the full range of the SFD clearance. A description of the rig and data acquisition process that is used to generate training data is presented. The construction of the recurrent neural network and training process is explained in detail. The robustness of the networks is tested over a wide range of validation data.

\section{TEST RIG DESCRIPTION}

Figure 1 shows a photograph of the test rig used for identification of the SFD and Figure 2 a schematic diagram. This test rig was used in [9] for the identification of the forward model of the SFD and in [5] for the preliminary work 
on the identification of the inverse model of the SFD. The rig is composed of an SFD bearing at one end and a shaft mounted on a self-aligning bearing at the other. With reference to Figure 2, the housing of the SFD (10) is mounted flexibly onto a frame (3) through a suspension (6) that comprises four flexible bars. The frame is bolted to a cast iron bedplate that is fixed to a concrete block.

The SFD (9) is oil film between the bearing housing (10) and the journal (8), which is the outer race of a rolling element bearing (see also Figure 3). Shaft rotation is irrelevant to the SFD input-output relationship since, in all rotordynamic applications, the SFD journal is prevented from rotating using dogs or retainer springs but allowed to orbit in the $x-y$ plane within the oil-filled clearance. For this reason, the shaft of the test rig was not rotated and controlled whirl motion of the journal within the oil-filled clearance was induced by excitation forces $P_{x}, P_{y}$ applied to the collar (5) from a pair of perpendicular shakers (Figures 1,2) that were driven by custom-generated input signals. The combined excitation provided by $P_{x}$ and $P_{y}$ is referred to as circular excitation since these forces were made to have equal and constant amplitudes and orthogonal phasing, thus mimicking rotational unbalance excitation.

As depicted in Figure 3, the SFD in this rig is a lightly sealed two-land bearing with a relatively deep groove. The
SFD journal is connected to the shaft by a roller bearing, as is typically the case. The journal rotation is prevented using dogs on the outer end-plate of the bearing housing that engage with dogs projecting from the outer end of the journal. Pressurised oil is supplied through three equi-spaced feed ports in the bearing housing. The dimensions of the SFD are presented in Table 1.

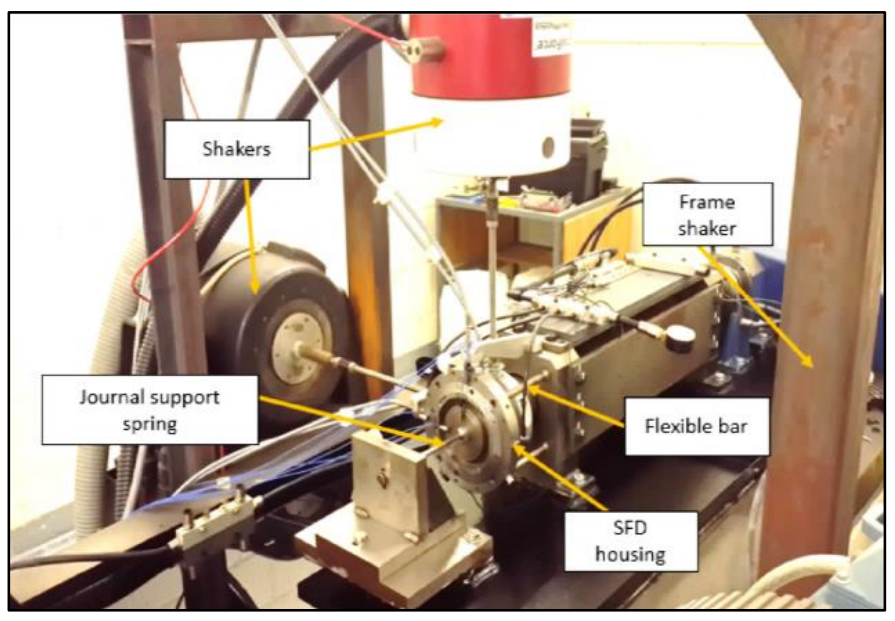

Figure 1: Photograph of the test rig

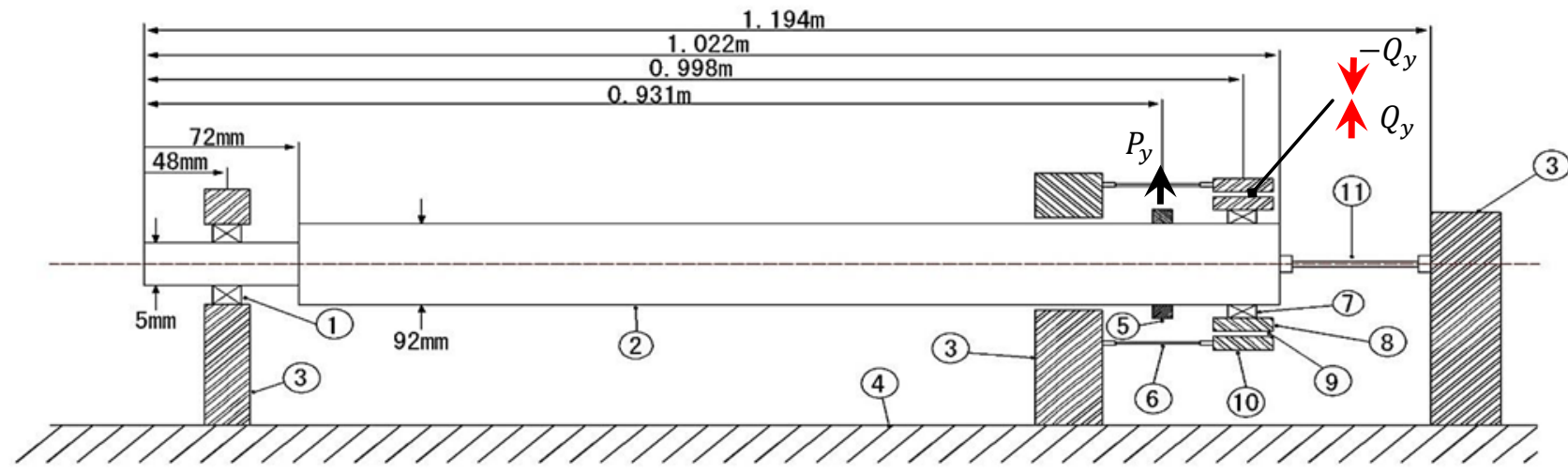

(1) Self-aligning ball bearing

(2) Shaft

(3) Frame

(4) Bedplate

(5) Shaker connection collar

(6) Housing support spring (flexible bar $\mathrm{x} 4$ )
(7) Roller bearing

(8) Damper journal $J$

(9) Oil annulus (SFD)

(10) Bearing housing $H$

(11) Journal support spring (flexible bar $\mathrm{x} 1$ )

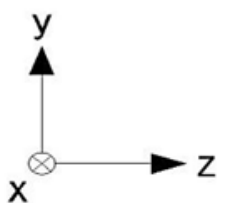

Figure 2: Test rig axial cut through (forces in $x$-direction not shown)

The instantaneous SFD forces on the journal in the $x, y$ directions are denoted by $Q_{x}, Q_{y}$. The corresponding forces on the bearing housing are therefore $-Q_{x},-Q_{y}$, as indicated in Figure 2 (where only the $y$-forces are shown). The displacements of the journal relative to the bearing housing in the $x, y$ directions are denoted by $x_{\mathrm{R}}, y_{\mathrm{R}}$ respectively. The identification procedure needs measurement of the input/output data sets (i.e. the time histories of $Q_{x}, Q_{y}$ respectively, and the corresponding time histories of $x_{\mathrm{R}}, y_{\mathrm{R}}$ respectively). The relative displacements $x_{R}, y_{R}$ are measured directly using proximity probes that are mounted on the bearing housing (10) and aimed at the shaft (the deformation of the rolling element bearing (7) is considered to be negligible). The SFD forces $Q_{x}, Q_{y}$ are determined using the displacement and acceleration of the bearing housing as well as the known modal data of the support structure subsystem (i.e. the bearing housing (10) mounted on the flexible suspension (6), Figure 2). The modal data were obtained by 
impact tests in [9] which showed that the dynamics of the support structure subsystem are accurately represented by a spring-mass model in each of the $x z$ and $y z$ for frequencies up to $800 \mathrm{~Hz}$. Hence, the SFD forces on the bearing housing $-Q_{x},-Q_{y}$ are given by:

$$
\begin{aligned}
& -Q_{x}=M_{\mathrm{H} x} \ddot{x}_{\mathrm{H}}+k_{\mathrm{H} x} x_{\mathrm{H}} \\
& -Q_{y}=M_{\mathrm{H} y} \ddot{y}_{\mathrm{H}}+k_{\mathrm{H} y} y_{\mathrm{H}}
\end{aligned}
$$

where, $M_{\mathrm{H} x}$ and $M_{\mathrm{H} y}$ are the effective mass, $k_{\mathrm{H} x}, k_{\mathrm{H} y}$ are the effective stiffness constants of the bearing housing in the $x z$ and $y z$ planes [9]. $x_{\mathrm{H}}, y_{\mathrm{H}}$ are the absolute Cartesian displacements of the bearing housing (10). These are measured by proximity probes mounted on the rigid frame (3) and aimed at (10). The corresponding accelerations $\ddot{x}_{\mathrm{H}}, \ddot{y}_{\mathrm{H}}$ are measured by accelerometers mounted on (10).

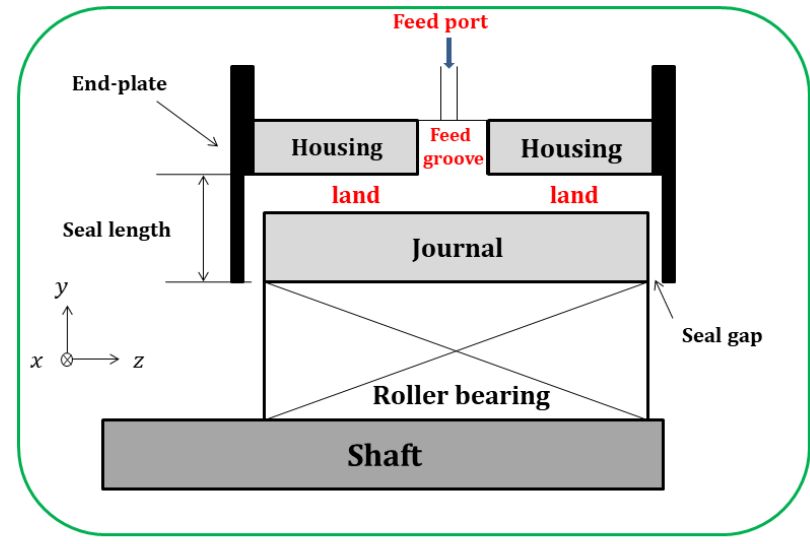

Figure 3: Test rig SFD cross-section

Table 1: Test rig SFD dimensions

\begin{tabular}{|c|c|}
\hline Journal radius & $69.8 \mathrm{~mm}$ \\
\hline Nominal radial clearance & $0.09 \mathrm{~mm}$ \\
\hline Land length & $6.23 \mathrm{~mm}$ \\
\hline Groove width & $7.52 \mathrm{~mm}$ \\
\hline Groove depth & $2.03 \mathrm{~mm}$ \\
\hline Seal gap & $0.5 \mathrm{~mm}$ \\
\hline Seal length & $3.15 \mathrm{~mm}$ \\
\hline Feed-port diameter & $1.5 \mathrm{~mm}$ \\
\hline Feed-port length & $25 \mathrm{~mm}$ \\
\hline
\end{tabular}

\section{EMPIRICAL-RNN INVERSE SFD MODEL}

As described earlier, the inverse model is fundamentally different from the forward model of the SFD since the latter is memoryless. This means that the instantaneous SFD forces $\mathbf{f}_{\mathbf{v}}=\left[\begin{array}{ll}Q_{x} & Q_{y}\end{array}\right]^{\mathrm{T}}$ can be determined given the instantaneous relative displacements $\mathbf{v}=\left[\begin{array}{ll}x_{\mathrm{R}} & y_{\mathrm{R}}\end{array}\right]^{\mathrm{T}}$ and velocities $\dot{\mathbf{v}}=$ $\left[\begin{array}{ll}\dot{x}_{\mathrm{R}} & \dot{y}_{\mathrm{R}}\end{array}\right]^{\mathrm{T}}$ :

$$
\mathbf{f}_{\mathbf{v}}=\mathbf{f}_{\mathbf{v}}(\mathbf{v}, \dot{\mathbf{v}})
$$

where $\mathbf{f}_{\mathbf{v}}$ is a nonlinear function of $\mathbf{v}, \dot{\mathbf{v}}$. In contrast, for the case of the inverse SFD model the instantaneous relative displacements/velocities (output) cannot be determined given only the instantaneous forces (input). However, a time history of $\mathbf{v}$ that corresponds to a given input time history of $\mathbf{f}_{\mathbf{v}}$ could be determined through the Nonlinear Autoregressive Exogenous (NARX) identification scheme used in time-series modelling [14], wherein the current value of the output $\mathbf{v}$ is determined from the previous values of the input $\mathbf{f}_{\mathbf{v}}$ and the previous values of the output $\mathbf{v}$ itself.

The NARX scheme is implemented by the RNN, as shown in Figures 4 and 5 . The signal $\mathbf{f}_{\mathbf{v}}\left(t_{k}\right)$ is the RNN's external input, where $t_{k}=(k-1) \Delta$ refers to the discrete times where $k=1,2, \ldots$, and $\Delta$ is the sampling time resolution. Normalisation is applied to the input data of the network, with a range of $[-1,1]$, where $\overbrace{(})$, refers to the normalised version of the variables in the network ( ) [14]. The RNN's output estimate of $\mathbf{v}\left(t_{k}\right)$ is denoted by $\breve{\mathbf{v}}\left(t_{k}\right)$ and its networknormalised form $\widetilde{\mathbf{v}}\left(t_{k}\right)$ is internally fed back to the RNN. The system memory is accounted for by the Tapped Delay Lines (TDLs in Figure 4), which contain multiple delays of the signals. The delayed signals $\widetilde{\mathbf{f}_{\mathbf{v}}}\left(t_{k-1}\right), \widetilde{\mathbf{f}_{\mathbf{v}}}\left(t_{k-2}\right), \ldots \widetilde{\tilde{f}_{\mathbf{v}}}\left(t_{k-n_{1}}\right)$ are carried by a multi-channel line (TDL no. 1 in Figure 5) in the respective $n_{1}$ channels, and the delayed signals $\widetilde{\mathbf{v}}\left(t_{k-1}\right)$, $\widetilde{\mathbf{v}}\left(t_{k-2}\right), \ldots \tilde{\widetilde{\mathbf{v}}}\left(t_{k-n_{1}}\right)$ are carried by TDL no. 2 in the respective $n_{2}$ channels (Figure 5).

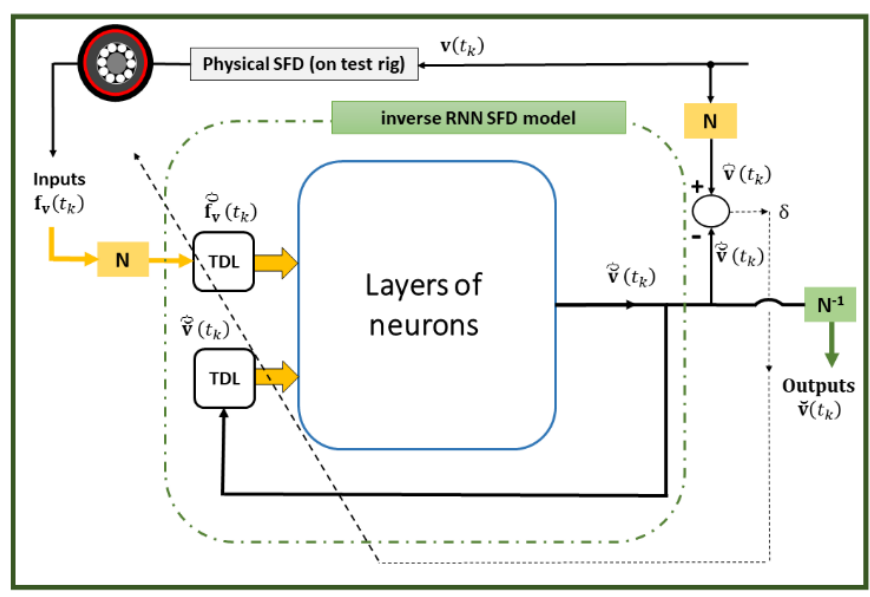

Figure 4: Inverse RNN SFD model with its identification procedure $\left(\mathrm{N}\right.$ : network normalisation; $\mathrm{N}^{-1}$ : de-normalisation; TDL: tapped delay line)

With reference to Figure 5, let $S^{(j)}$ be the number of neurons in the $j^{\text {th }}$ layer, $J$ be the total number of layers and $n_{1}, n_{2}$ be 
the numbers of delays in TDL no.1 and TDL no.2 respectively. If $\mathbf{a}_{k}^{(j)}$ is the $S^{(j)} \times 1$ vector comprising the signal outputs of the $j^{\text {th }}$ layer, then (Torres-Cedillo and Bonello [5]):

$$
\begin{aligned}
& \mathbf{a}_{k}^{(J)}=\widetilde{\mathbf{v}}\left(t_{k}\right) \\
& \mathbf{a}_{k}^{(1)}=\mathbf{g}^{(1)}\left(\mathbf{W}^{(1)} \mathbf{p}_{k}+\mathbf{b}^{(1)}\right) \\
& \mathbf{a}_{k}^{(j)}=\mathbf{g}^{(j)}\left(\mathbf{W}^{(j)} \mathbf{a}_{k}^{(j-1)}+\mathbf{b}^{(j)}\right), j=2, \ldots J
\end{aligned}
$$

where: $\mathbf{p}_{k}$ be the $\left(2 n_{1}+2 n_{2}\right) \times 1$ column matrix comprising the signal inputs to layer no. 1 :

$$
\mathbf{p}_{k}=\left[\begin{array}{llllll}
\tilde{\mathbf{f}}_{\mathbf{v}}\left(t_{k-1}\right)^{\mathrm{T}} & \ldots & \tilde{\mathbf{f}}_{\mathbf{v}}\left(t_{k-n_{1}}\right)^{\mathrm{T}} & \tilde{\widetilde{\mathbf{v}}}\left(t_{k-1}\right)^{\mathrm{T}} & \ldots & \widetilde{\mathbf{v}}\left(t_{k-n_{2}}\right)^{\mathrm{T}}
\end{array}\right]^{\mathrm{T}}
$$

$$
\widetilde{\mathbf{f}}_{\mathbf{v}}\left(t_{k-n}\right), \widetilde{\widetilde{\mathbf{v}}}\left(t_{k-n}\right)=\mathbf{0}, \quad t_{k-n}<t_{1}
$$

$\mathbf{W}^{(j)}, \mathbf{b}^{(j)}$ are respectively the matrix of weights and vector of biases of the $j^{\text {th }}$ layer and $\mathbf{g}^{(j)}(\quad)$ is a vector operator comprising the transfer functions of the neurons of the $j^{\text {th }}$ layer.

Figure 4 also shows the identification/training procedure with the parameters of the proposed network architecture, which are optimised to minimise the error-difference $\delta$ between the output of the network $\widetilde{\mathbf{v}}\left(t_{k}\right)$ and the actual data (i.e. target) output $\tilde{\mathbf{v}}\left(t_{k}\right)$.

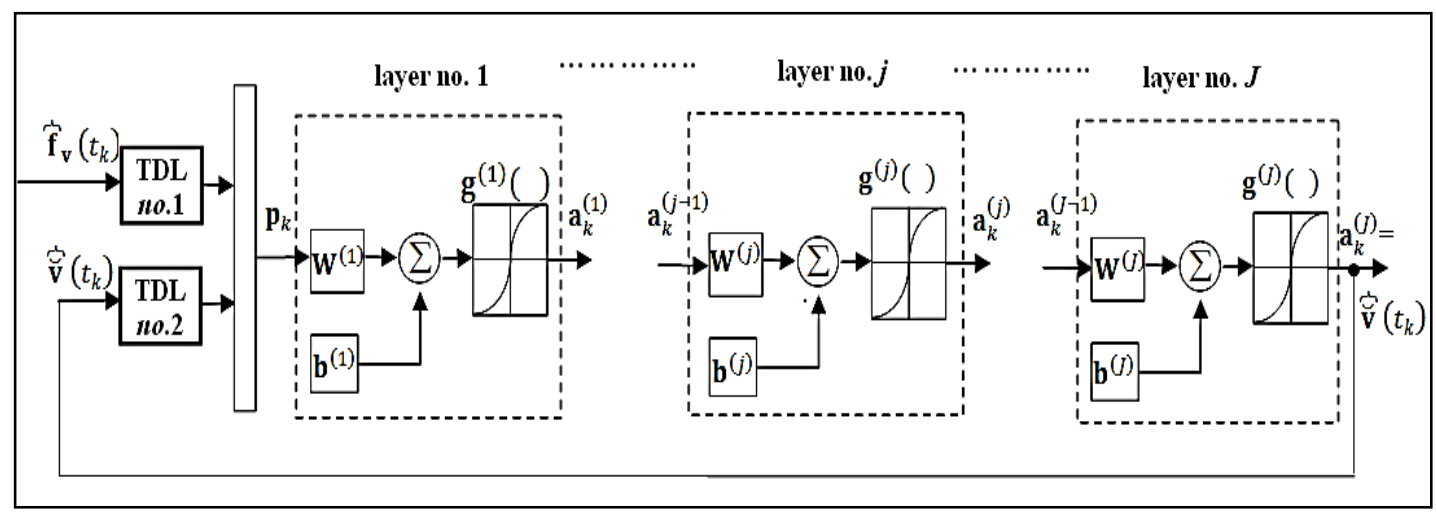

Figure 5: Architecture of the RNN in Figure 4 [5]

\subsection{GENERATION OF INPUT/OUTPUT TRAINING DATA FOR SFD IDENTIFICATION}

Sets of empirical data have been measured to be employed as training data of RNN. These data sets were obtained under conditions created by inputting orthogonally phased chirp signals, with constant amplitude and steadily increasing frequency, to the amplifiers of the two orthogonal shakers acting at the collar (5) (Figure 2). The circular chirp excitation at the collar is therefore described as follows:

$$
\begin{gathered}
P_{x}(t)=A \cos \left(2 \pi\left\{f_{\text {init }} t+\frac{\Delta f}{\Delta t}\left(\frac{t^{2}}{2}\right)\right\}\right) ; \\
P_{y}(t)=-A \sin \left(2 \pi\left\{f_{\text {init }} t+\frac{\Delta f}{\Delta t}\left(\frac{t^{2}}{2}\right)\right\}\right)
\end{gathered}
$$

where $\quad \frac{\Delta f}{\Delta t}=\frac{f_{\text {end }}-f_{\text {init }}}{t_{\text {end }}-t_{\text {init }}}$

and $A$ is a constant excitation amplitude, while the subscripts end and init are employed to identify the final and initial fixed values of excitation frequencies and time, respectively. The supply pressure was maintained at 0.8 bar throughout testing. The oil used was Shell Morlina 5 and was maintained at a temperature $29^{\circ} \mathrm{C}$.
Four sets of training data were generated from four separate frequency sweeps $10-100 \mathrm{~Hz}$ at shaker force amplitudes (as measured by the force gauges) of 20,50, 80, $100 \mathrm{~N}$ respectively (i.e. these are the amplitudes of $P_{x}, P_{y}$ in eq. (7)) The training data consists of the input training data (SFD forces $Q_{x}, Q_{y}$, estimated according to eqs. $(1 \mathrm{a}, \mathrm{b})$ ) and the corresponding output (target) training data (measurements of the relative motion Cartesian components $x_{R}, y_{R}$ ). Consequently, four networks (RNNs) were identified for the inverse SFD model, one for each of the four sets of training data. Figure 6 shows the training data gathered at the frequency sweep with $20 \mathrm{~N}$ shaker force amplitude (eq. (7)).

\subsection{RNN ARCHITECTURE AND TRAINING}

In total, 32,173 training pairs were used to train the network. Each of the four sets of empirical data was applied to the RNN, whose generic architecture is shown in Figure 4. The RNN design and training were implemented using MATLAB 2017a through the Neural Network Toolbox [14]. In this research, various architectures of the RNN were tested by applying three layers with different numbers of neurons, with $n_{1}=2$ delays for the input and $n_{2}=2$ for the feedback. Tansigmoid transfer functions $[5,14]$ were used for the neurons, except for those in the output layer, which were linear transfer functions. The training data sets (inputs and outputs) were 
divided randomly into three subsets: training set (60\%), testing set $(20 \%)$, and validation set $(20 \%)$ (this latter set is used by the Neural Network Toolbox [14] within the training process and should not be confused with the post-training validation tests described in the following Section 4). The LevenbergMarquardt optimisation method [14] training procedure was used in order to optimise the weights and biases of the network (eqs. $(4 a, b)$ ). With reference to Figure 4 , the goal of the optimisation was the convergence of the mean square value of the error $\delta$ (MSE) to a value below a set tolerance specification. With reference to Figure 5, the number of layers used was 3 (including the output layer). A lower number of layers did not yield satisfactory convergence. The number of neurons of the output layer was fixed at 2 since the output channel contains two signals $\left(x_{\mathrm{R}}, y_{\mathrm{R}}\right)$. The respective numbers of neurons in the first two layers ("hidden layers") were determined using an exhaustive search method recently introduced by [15]. In this method, the number of neurons in each hidden layer was progressively increased, starting from a few neurons, and, for each trial architecture, the RNN was trained 10 times. Due to the random initialization of the weights and biases, training multiple times for a given trial architecture is considered necessary to avoid the MSE being trapped in local minima [15]. The optimal architecture was then identified as the one that gave the lowest MSE. For the training data in Figure 6 (gathered at shaker force amplitudes of $20 \mathrm{~N}$ ), the optimal architecture consisted of $8,7,2$ neurons for first, second, and third layer, respectively.

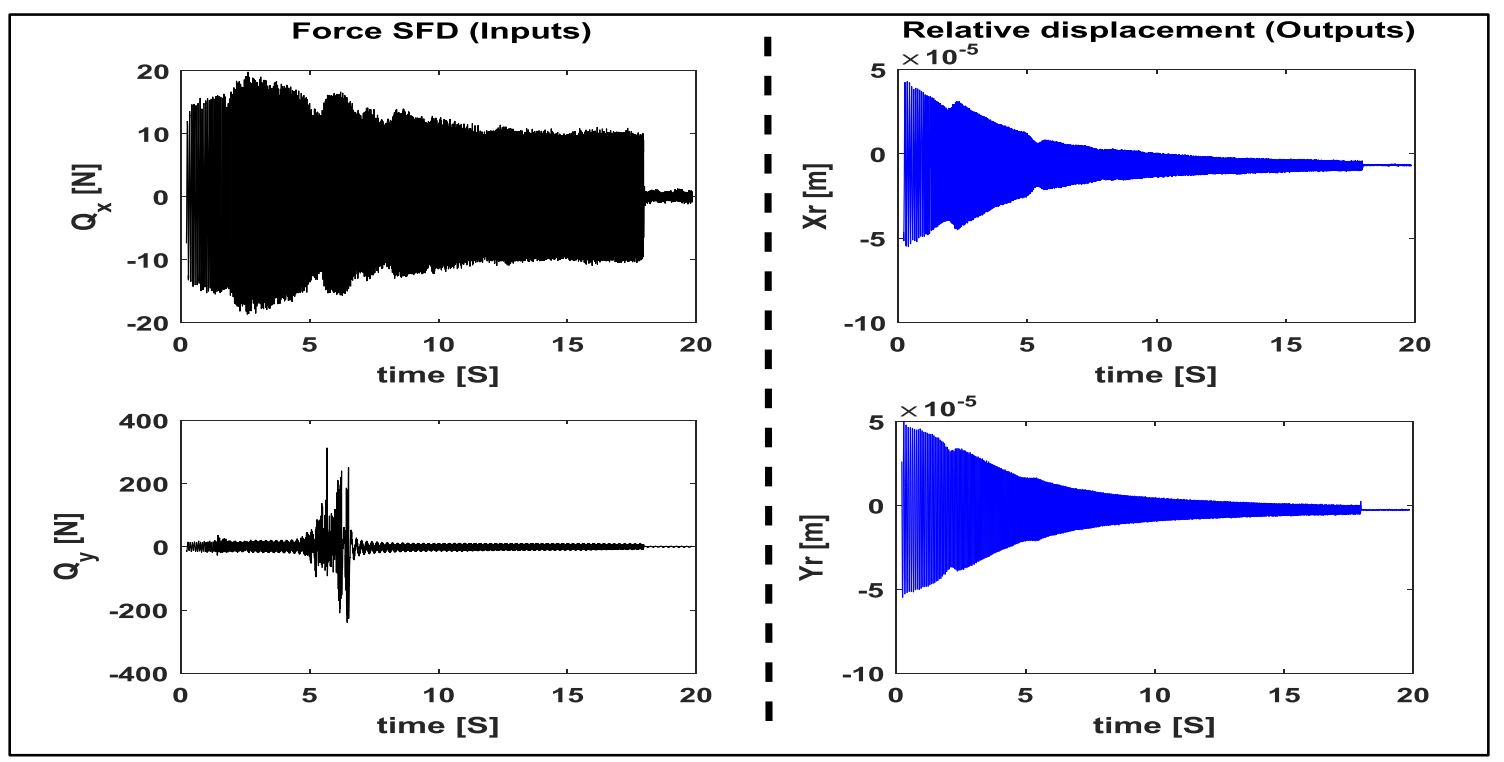

Figure 6: Training data (input/output) gathered using chirp excitation spanning 10-100 Hz at amplitude $20 \mathrm{~N}$ (eq. (7))

\section{VALIDATION TESTS}

The trained networks were tested to predict the time histories of the journal displacements $x_{\mathrm{R}}, y_{\mathrm{R}}$ under conditions that were vastly different from those they were subjected to in training. The validation tests presented in this section were conducted using shaker signals $P_{x, y}(t)$ that provided circular excitation at a single discrete frequency (i.e. $\Delta f=0$ in eq. (7)). The tests considered discrete amplitudes in the range 25 to $105 \mathrm{~N}$ and discrete frequencies in the range 35 to $105 \mathrm{~Hz}$. On the other hand, the corresponding predictions for $x_{R}, y_{R}$ for these conditions are provided by the RNN that was trained with shaker signals $P_{x, y}(t)$ that provided circular chirp excitation (continuously varying frequency i.e. $\Delta f \neq 0$ in eq. (7)), spanning $10-100 \mathrm{~Hz}$ at a single fixed amplitude of $20 \mathrm{~N}$ (Figure 6).

Figure 7 refers to the validation tests at an excitation force $P_{x, y}(t)$ of $95 \mathrm{~N}$ amplitude and $55 \mathrm{~Hz}$ frequency. The first column of Figure 7 shows the time histories of the SFD forces $Q_{x, y}$ (computed from eqs. (1a,b)). These are employed as inputs to the trained $\mathrm{RNN}$, and its outputs (which are its estimates for the time histories of the SFD relative displacements $\left.x_{\mathrm{R}}(t), y_{\mathrm{R}}(t)\right)$ are compared with the actual measurements in the second column of Figure 7. Also, the steady state trajectory (orbit) plots on the SFD journal relative to the bearing housing, i.e. $y_{R}$ vs $x_{R}$, are shown in the third column of the Figure. Notice that measured clearance boundary of the SFD, which is nominally a circle of radius $0.09 \mathrm{~mm}$, has a distorted triangular form as a result of machining irregularity. This clearance boundary is the measured trajectory $\left(y_{R}\right.$ vs $\left.x_{R}\right)$ obtained when the journal is gently brushed against the housing bore with no oil in the clearance (by applying shaker forces of suitably high amplitude and very low frequency).

Figure 7 shows that the RNN prediction for $x_{\mathrm{R}}(t), y_{\mathrm{R}}(t)$ correlates very well with the measurement. Figure 8 compares the fast Fourier transforms (FFTs) of the relative displacements of the measured and RNN-predicted relative displacement signals $x_{\mathrm{R}}, y_{\mathrm{R}}$ in Figure 7 . As can be seen, the fundamental frequency of the signals is accurately predicted. The discrepancies at $0 \mathrm{~Hz}$ for the $x$-vibration spectrum reflect slight differences in the mean value (also evident from the upper graph of the middle column of Figure 7). The FFT of 
the $y$-vibration in Figure 8 shows that the RNN also predicts a faint second harmonic which is not observed in the measurement.

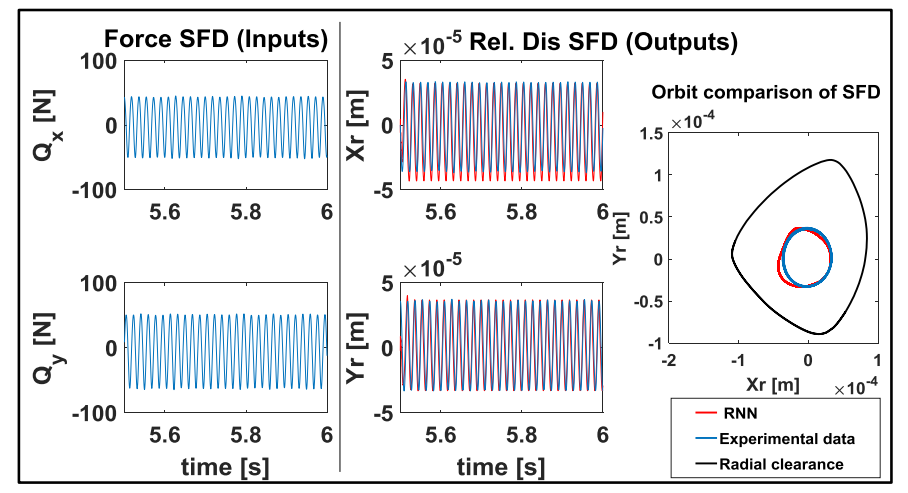

Figure 7: Validation tests of the inputs/outputs network under circular excitation amplitude/frequency of $95 \mathrm{~N} / 55 \mathrm{~Hz}$; (SFD forces, relative displacements time histories \& orbits)

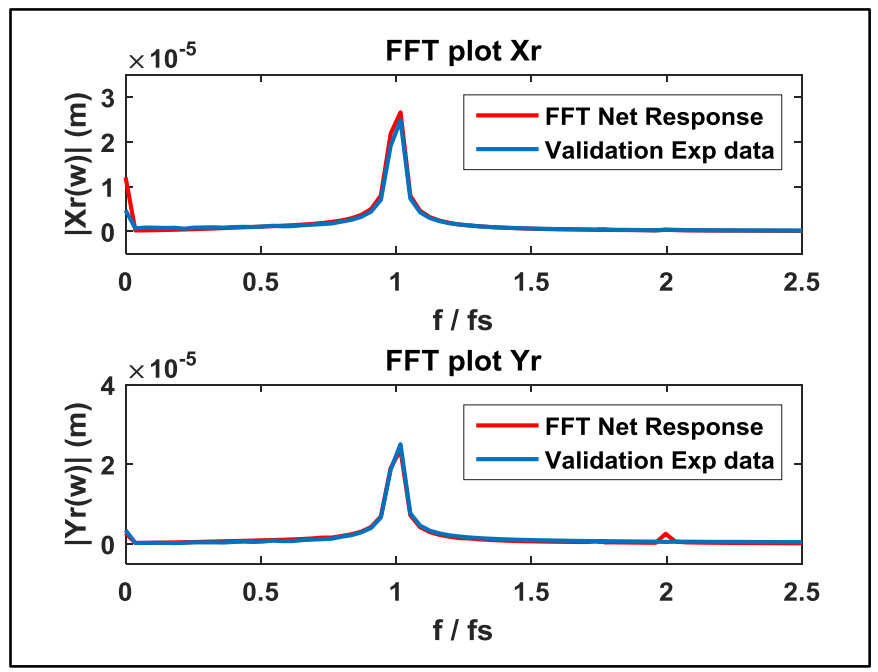

Figure 8: Comparison between the FFTs of measured and predicted relative displacements $\left(x_{\mathrm{R}}, y_{\mathrm{R}}\right)$ under circular excitation amplitude/frequency of $95 \mathrm{~N} / 55 \mathrm{~Hz}$

Figures 9-16 present validation test results for different excitation amplitude/frequency combinations. These results show that, for a given amplitude of shaker excitation, the amplitudes of $x_{R}, y_{R}$ decrease with increasing frequency for both measurements and RNN predictions (e.g. comparing Figure 9 with Figure 10 or Figure 11 with Figure 12). The accuracy of the amplitude prediction is observed to be less satisfactory for the low excitation force amplitude of $25 \mathrm{~N}$ (Figures 9, 10), then it is for the higher excitation force amplitudes 55-105 N (Figures 11-16). This is somewhat surprising since the RNN used in Figures 7-16 was trained at a constant amplitude of $20 \mathrm{~N}$. Nonetheless, in all cases, the RNN-predicted time histories of $x_{R}, y_{R}$ in Figure 9-16 correctly track the fundamental frequency of the measured time histories, as can be from the FFTs of Figures 17 and 18, which correspond to the worst cases of amplitude prediction i.e. Figures 9 and 10 respectively. Figures 17 and 18 show a discrepancy in the $0 \mathrm{~Hz}$ frequency component, which corresponds to the difference in the mean values of $x_{R}, y_{R}$ that is evident from the middle columns of Figures 9 and 10. The resulting difference in the mean positions of the predicted and measured orbits within the clearance (third columns of Figures 9 and 10) appears to be linked to the discrepancy in the amplitude prediction, possibly mirroring the fact that the journal vibration of a physical SFD is sensitive to its position within the clearance. However, it should be emphasised that neural networks are a "black box" identification scheme, and so it is difficult to attribute physical meaning to discrepancies between their output and that of the physical system they are trained to simulate.

Despite these discrepancies, the predictions obtained are, generally speaking, on a par with those in [5], which were obtained with considerably more training effort. In certain cases they are considerably superior, as evident by comparing the result for $105 \mathrm{~N} / 65 \mathrm{~Hz}$ obtained with the present RNN (Figure 16), with that obtained by the RNN of [5], which is reproduced in Figure 19.

The reduction in training effort using the approach of this paper, relative to the previous approach [5] is quantified as follows:

- Effort to generate experimental training data is reduced by a factor of 195. In reference [5], the authors generated training data for 13 discrete amplitude cases $(25-150 \mathrm{~N})$ and for each of these, 15 discrete frequency cases (10-150 $\mathrm{Hz}$ ), resulting in 195 experimental runs to generate the training data. With the current approach, all these runs are replaced by a single experimental run using a chirp in the range $10-100 \mathrm{~Hz}$ at fixed amplitude of $20 \mathrm{~N}$.

- Effort to train the RNNs is reduced by a factor of 2. In reference [5], the authors had to train two networks to cover the high and low-frequency cases. The present approach gives one network to cover all cases of high and low frequencies.

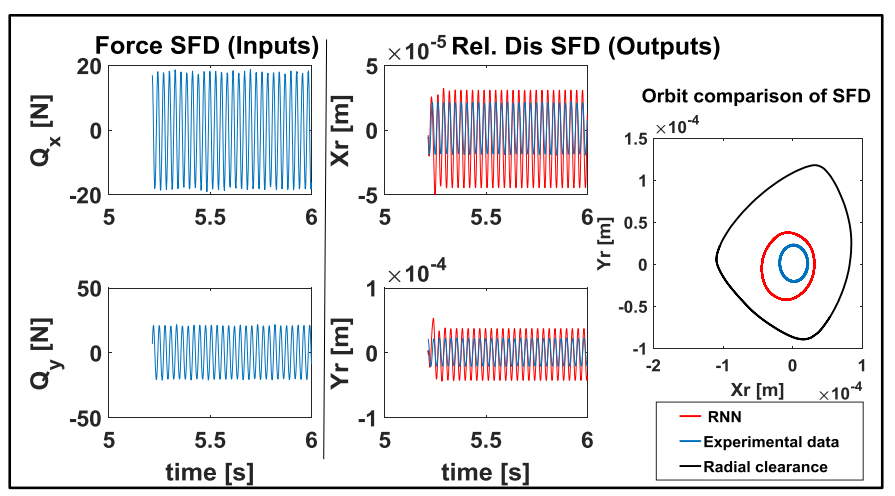

Figure 9: Validation tests of the inputs/outputs network under circular excitation amplitude/frequency of $25 \mathrm{~N} / 35 \mathrm{~Hz}$; (SFD forces, relative displacements time histories \& orbits) 


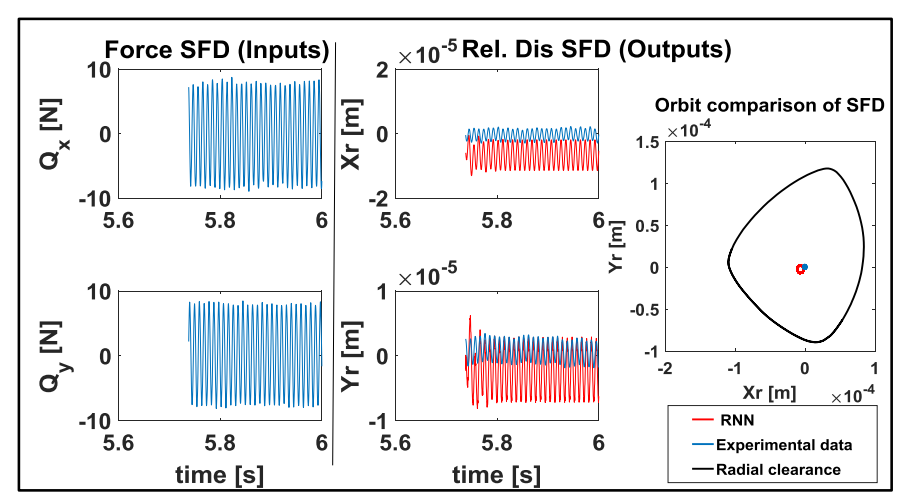

Figure 10: Validation tests of the inputs/outputs network under circular excitation amplitude/frequency of $25 \mathrm{~N} / 105 \mathrm{~Hz}$; (SFD forces, relative displacements time histories \& orbits)

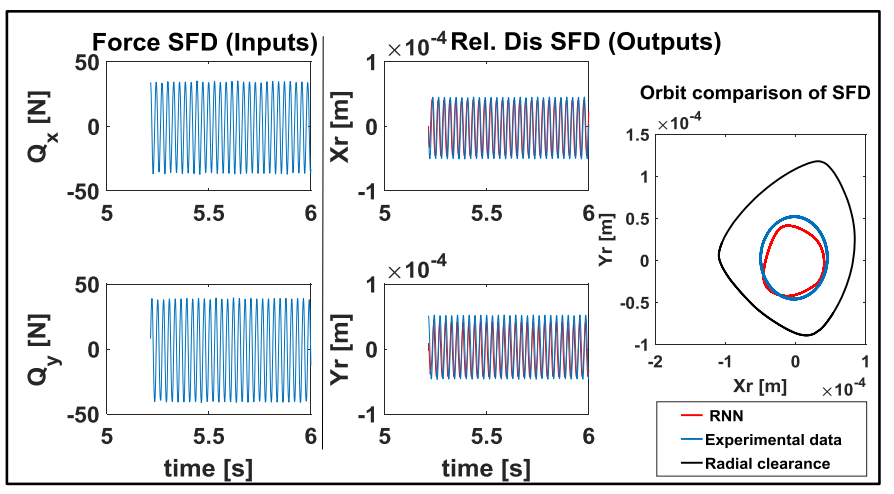

Figure 11: Validation tests of the inputs/outputs network under circular excitation amplitude/frequency of $55 \mathrm{~N} / 35 \mathrm{~Hz}$; (SFD forces, relative displacements time histories \& orbits)

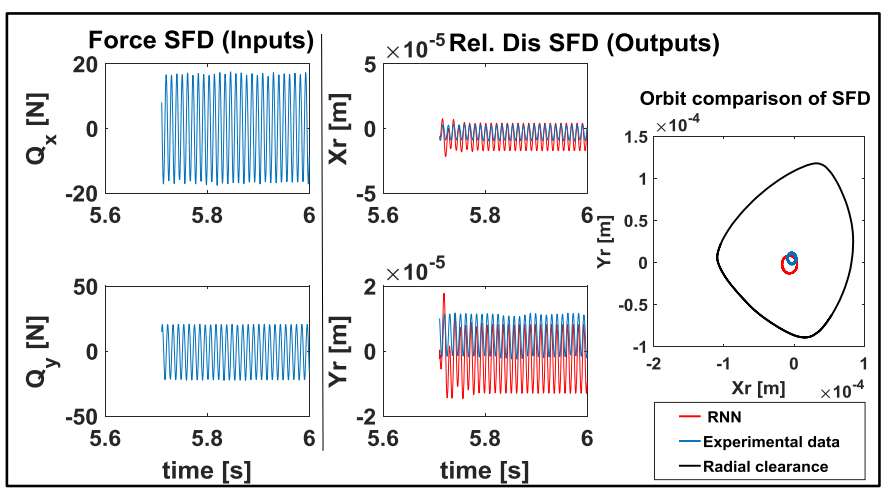

Figure 12: Validation tests of the inputs/outputs network under circular excitation amplitude/frequency of $55 \mathrm{~N} / 95 \mathrm{~Hz}$; (SFD forces, relative displacements time histories \& orbits)

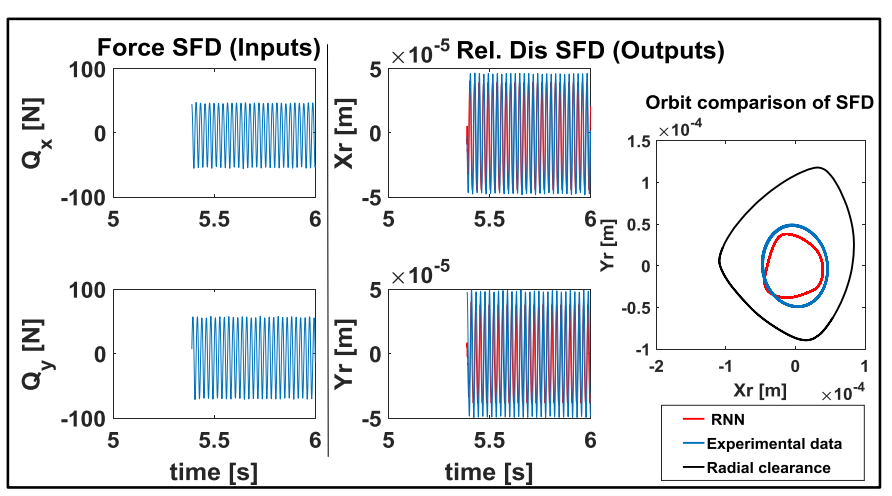

Figure 13: Validation tests of the inputs/outputs network under circular excitation amplitude/frequency of $95 \mathrm{~N} / 45 \mathrm{~Hz}$; (SFD forces, relative displacements time histories \& orbits)

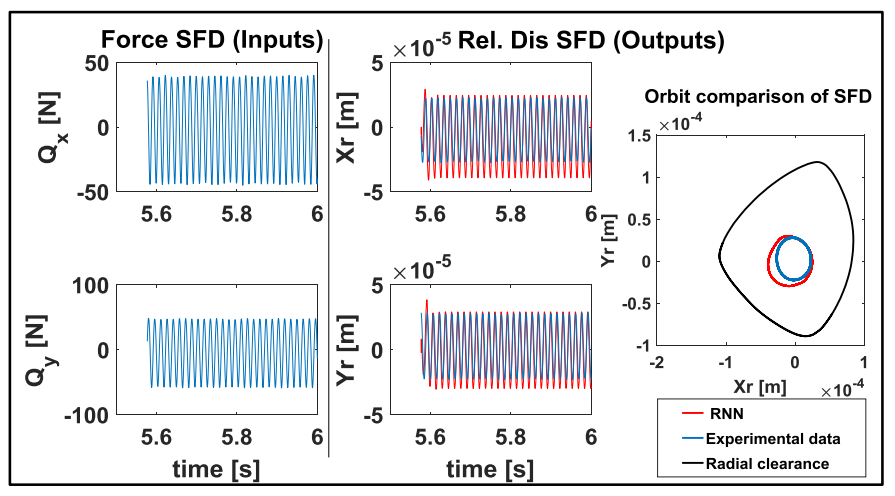

Figure 14: Validation tests of the inputs/outputs network under circular excitation amplitude/frequency of $95 \mathrm{~N} / 65 \mathrm{~Hz}$; (SFD forces, relative displacements time histories \& orbits)

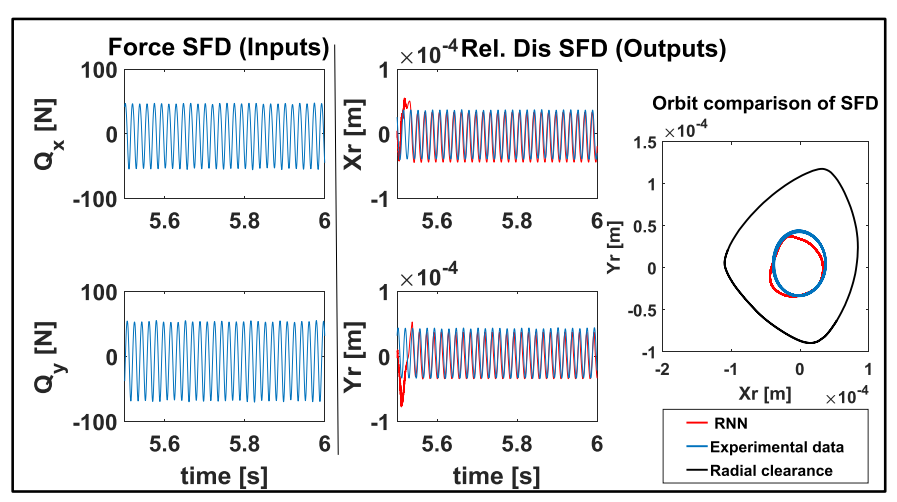

Figure 15: Validation tests of the inputs/outputs network under circular excitation amplitude/frequency of $105 \mathrm{~N} / 55 \mathrm{~Hz}$; (SFD forces, relative displacements time histories \& orbits) 


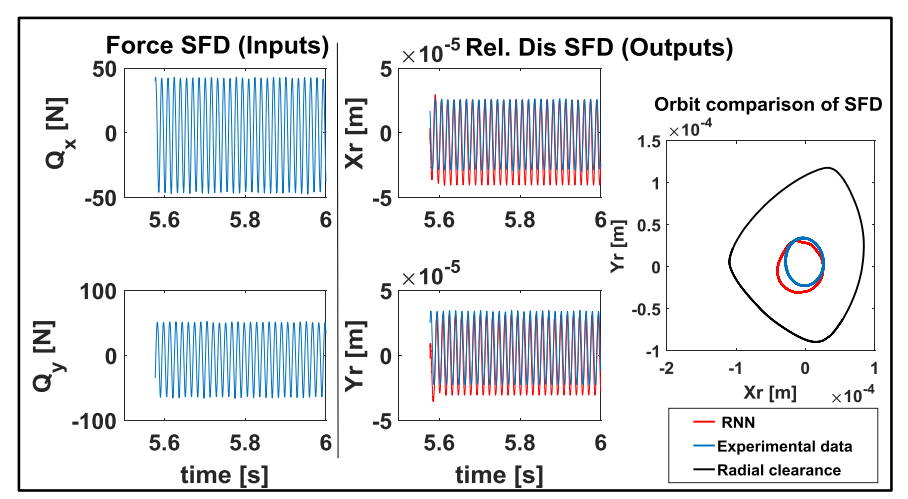

Figure 16: Validation tests of the inputs/outputs network under circular excitation amplitude/frequency of $105 \mathrm{~N} / 65 \mathrm{~Hz}$; (SFD forces, relative displacements time histories \& orbits)

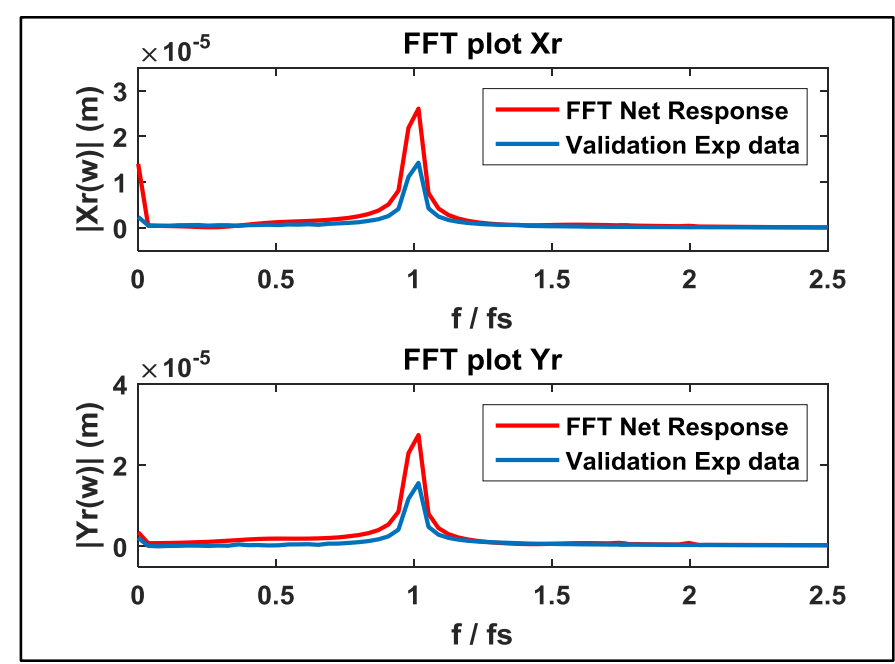

Figure 17: Comparison between the FFTs of measured and predicted relative displacements $\left(x_{\mathrm{R}}, y_{\mathrm{R}}\right)$ under circular excitation amplitude/frequency of $25 \mathrm{~N} / 35 \mathrm{~Hz}$

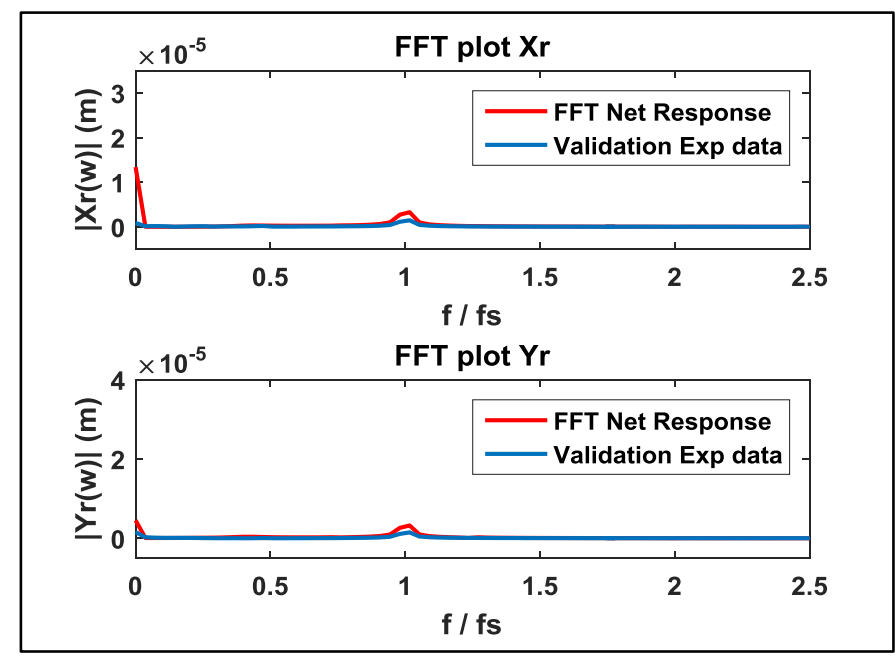

Figure 18: Comparison between the FFTs of measured and predicted relative displacements $\left(x_{\mathrm{R}}, y_{\mathrm{R}}\right)$ under circular excitation amplitude/frequency of $25 \mathrm{~N} / 105 \mathrm{~Hz}$

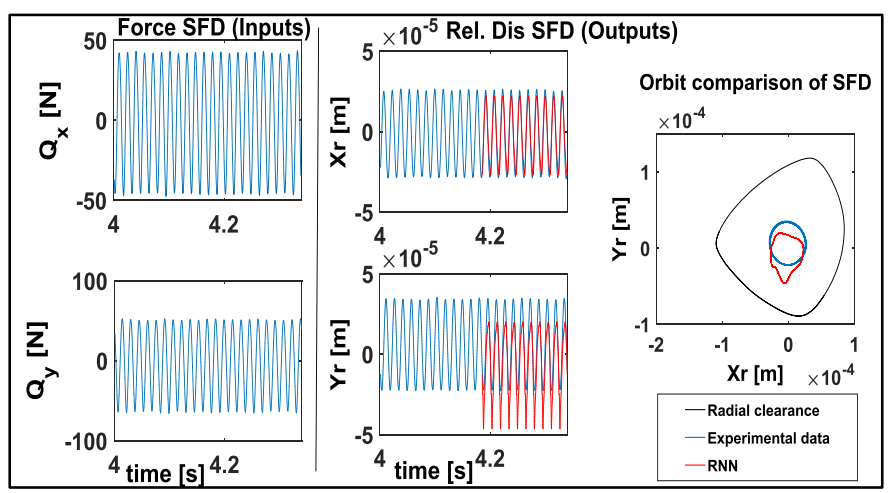

Figure 19: Validation tests of the inputs/outputs network under circular excitation amplitude/frequency of $105 \mathrm{~N} / 65 \mathrm{~Hz}$; (SFD forces, relative displacements time histories \& orbits)[5]

\section{CONCLUSIONS}

The aim of this investigation was to present an improved non-parametric empirical model of the inverse SFD dynamics. An RNN was trained to predict the time histories of the relative displacements (outputs) from the time histories of the SFD forces (inputs). The RNN accounts for system memory using the NARX scheme that considers the current value of the output as a function of the past values of both the input and the output. The test rig was specifically designed to acquire SFD bearing force and relative displacement time histories by simultaneously applying orthogonal forces to generate excitation. The training data for the RNNs were generated by exciting the rig with chirp signals. Different topologies of the RNN were trained by considering different numbers of layers and numbers of neurons in an exhaustive search method to identify an optimal architecture.

The best-obtained network architecture can predict experimental data within satisfactory accuracy when subjected to excitation conditions that were vastly different from those used for training (the post training validation tests being conducted at considerably higher amplitude and fixed frequency). Also, its output correctly tracks the fundamental frequency of the input, as checked by performing Fourier analysis. In comparison to an earlier method, the proposed methodology is shown to produce RNNs with less training effort that can offer superior quality predictions. The results show that the RNN inverse SFD model in this work has the potential of serving as a reliable virtual instrument for estimating the journal displacement as part of the solution of the inverse rotordynamic problem for unbalance identification.

\section{NOMENCLATURE}

$\mathbf{a}_{k}^{(j)} \quad$ vector of the signal outputs of the $j$ th

neural network layer, Sec. 3

b neural network bias vector, Sec. 3

$\mathrm{f}_{\text {init }} \quad$ initial shaker excitation frequency $(\mathrm{Hz})$

$\mathrm{f}_{\text {end }} \quad$ finial shaker excitation frequency $(\mathrm{Hz})$

$\mathbf{f}_{\mathbf{v}} \quad$ SFD force vector 


\begin{tabular}{|c|c|}
\hline $\mathbf{g}^{(j)}$ & $\begin{array}{l}\text { vector of neural network activation } \\
\text { functions for layer no. } j \text {, Sec. } 3\end{array}$ \\
\hline$j$ & layer number of the network, Sec. 3 \\
\hline$J$ & $\begin{array}{l}\text { total number of layers within the network, Sec. } 3 \\
\text { effective masses of the support }\end{array}$ \\
\hline$M_{\mathrm{H} x}, M_{\mathrm{H} y}$ & $\begin{array}{l}\text { structure in the } x \text { and } y \text { directions } \\
\text { respectively }(\mathrm{kg})\end{array}$ \\
\hline $\mathrm{N}$ & network normalization, Figure 4 \\
\hline $\mathrm{N}^{-1}$ & reverse normalization, Figure 4 \\
\hline $\mathbf{p}_{k}$ & $\begin{array}{l}\text { the column matrix comprising the signal inputs } \\
\text { to layer no. } 1 \text { of network. Sec. } 3\end{array}$ \\
\hline $\begin{array}{l}P_{x}(t) \\
P_{y}(t)\end{array}$ & $\begin{array}{l}\text { shaker forces simultaneously applied } \\
\text { at the collar in } x \text { and } y \text { directions }(\mathrm{N})\end{array}$ \\
\hline$Q_{x}, Q_{y}$ & $\begin{array}{l}\text { squeeze-film damper forces in } x \text { and } y \\
\text { directions }(\mathrm{N})\end{array}$ \\
\hline$S^{(j)}$ & number of neurons in the layer $j^{\text {th }}$, Sec. 3 \\
\hline$t$ & time $(s)$ \\
\hline$t_{k}$ & discrete time (s) \\
\hline & $=\left[\begin{array}{ll}x_{\mathrm{R}} & y_{\mathrm{R}}\end{array}\right]^{\mathrm{T}}$ i.e. vector of the \\
\hline $\mathbf{v}$ & $\begin{array}{l}\text { instantaneous Cartesian displacements } \\
\text { of journal relative to housing }\end{array}$ \\
\hline $\mathbf{W}^{(\mathrm{j})}$ & $\begin{array}{l}\text { matrix of network weights at } j \text { th layer } \\
\text { neural network. Sec. } 3\end{array}$ \\
\hline$x_{\mathrm{R}}, y_{\mathrm{R}}$ & $\begin{array}{l}x, y \text { displacements of the SFD journal } \\
\text { centre relative to bearing housing } \\
\text { centre }(\mathrm{m})\end{array}$ \\
\hline$\delta$ & $\begin{array}{l}\text { error between the network output and } \\
\text { training target. Sec. } 3\end{array}$ \\
\hline ) & $\begin{array}{l}\text { Network prediction for the variable } \\
(\text { ) }\end{array}$ \\
\hline$\tau$ & $\begin{array}{l}\text { Network-normalised version of the } \\
\text { variable ( ) }\end{array}$ \\
\hline
\end{tabular}

\section{ACKNOWLEDGMENTS}

The authors are grateful to The Higher Committee for Education Development in Iraq for financially supporting the current research.

\section{REFERENCES}

[1] D. Peters, R. Nordmann, B. Domes, P. Maass, "Inverse Determination of Imbalance Distributions", in: Eighth International Conference on Vibrations in Rotating Machinery 7-9 September 2004, University of Wales, Swansea, UK, IMechE Conference Transactions 2004-2, 521-530.

[2] V. Dicken, P. Maaß, I. Menz, J. Niebsch, R. Ramlau, "Nonlinear inverse unbalance reconstruction in rotor dynamics", Inverse Problems in Science and Engineering. 13 (2005) 507-543.

[3] R. Ramlau, V. Dicken, P. Maaß, C. Streller, A. Rienäcker, "Inverse imbalance reconstruction in rotordynamics", ZAMM - Journal of Applied Mathematics and Mechanics / Zeitschrift Für Angew. Math. Und Mech. 86 (2006) 385-399.

[4] S.G.T. Cedillo, P. Bonello, "An equivalent unbalance identification method for the balancing of nonlinear squeeze-film damped rotordynamic systems", Journal of Sound and Vibration. 360 (2016) 53-73.

[5] S.G. Torres Cedillo, P. Bonello, "Empirical identification of the inverse model of a squeeze-film damper bearing using neural networks and its application to a nonlinear inverse problem", Journal of Vibration and Control. (2016) 1-22.

[6] A.W. Lees, J.K. Sinha, M.I. Friswell, "The Identification of the Unbalance of a Flexible Rotating Machine From a Single Rundown", Journal of Engineering for Gas Turbines and Power. 126 (2004) 416.

[7] M. Villafane Saldarriaga, V. Steffen, J. Der Hagopian, J. Mahfoud, "On the balancing of flexible rotating machines by using an inverse problem approach", Journal of Vibration and Control 17 (2011) 10211033.

[8] C-C. Siew, M. Hill, R. Holmes, M. Brennan, "A theoretical and experimental investigation into the vibration response of a flexible rotor and squeeze-film damper assembly", Proceedings of the IMechE Part C. 215 (2001) 1251-1269.

[9] K.H. Groves, P. Bonello, "Empirical identification of squeeze-film damper bearings using neural networks", Mechanical Systems and Signal Processing. 35 (2013) 307-323.

[10] K.H. Groves, P. Bonello, P.M. Hai, "Efficient dynamic analysis of a whole aeroengine using identified nonlinear bearing models", Proceedings of the Institution of Mechanical Engineers, Part C: Journal of Mechanical Engineering Science. 226 (2011) 66-81.

[11] F.A. Rodrigues, F. Thouverez, C. Gibert, L. Jezequel, "Chebyshev polynomials fits for efficient analysis of finite length squeeze film damped rotors", Journal of Engineering for Gas Turbines and Power. 125 (2003) 175-183.

[12] Z. Chen, Y. Jiao, S. Xia, W. Huang, Z. Zhang, "An efficient calculation method of nonlinear fluid film forces in journal bearing", Tribology Transactions. 45 (2002) 324-329.

[13] M.F. Bin Hassan, P. Bonello, "A neural network identification technique for a foil-air bearing under variable speed conditions and its application to unbalance response analysis", Journal of Tribology. 139 (2017) 1-13.

[14] M.H. Beale, M.T. B.Hagan, H.B. and Demuth, "Neural Network Toolbox" User's Guide R2017a, MathWorks, Inc., n.d.

[15] B.R. Al-Kaseem, H.S. Al-Raweshidy, A.-D. Yousif, K. Banitsas, "A New Intelligent Approach for Optimising 6LoWPAN MAC Layer Parameters", IEEE Access Journal. 5 (2017) 1-11. 